\title{
NLTE ANALYSIS OF THE WOLF-RAYET STAR HD193077 (WN5+abs)
}

\author{
W. Schmutz, W.-R. Hamann, U. Wessolowski \\ Institut für Theoretische Physik und Sternwarte der Universität Kiel \\ 01 shausenstrasse, 2300 Kiel 1, Federal Republic of Germany
}

A model atmosphere code that accounts for the special physical conditions in WolfRayet atmospheres (Hamann and Schmutz, 1986; Wessolowski et al., 1987) is used to analyse the spectrum of the Wolf-Rayet star HD193077 (WN5+abs). The stellar parameters are determined such that the profiles of the helium lines He I $\lambda \lambda 4471,5876$, He II $\lambda 5412$, and the absolute visual magnitude are reproduced.

In order to estimate the systematic errors introduced by the model assumptions, we perform some test calculations. Instead of the velocity-law exponent of $\beta=1$, another fit is obtained with $B=0.5$ (Fig. 1). This fit yields an effective temperature of about $3000 \mathrm{~K}$ higher than with $B=1$. In order to simulate the effect of the suspected hydrogen content, a further fit (Fig. 2) is made when one free electron per helium atom is added artificially. This has only marginal influence on the derived temperature $(+100 \mathrm{~K})$. Thus, we conclude that our model assumptions may introduce a systematic error of the order of 5000K.

The best agreement with the line profiles (Fig. 2) is obtained with

$$
\begin{aligned}
T_{\text {eff }} / K & =33600 \pm 200 \quad \text { (for systematic errors see text) } \\
R_{\star} / R_{\odot} & =15 \pm 3 \\
\log \left(L / L_{\odot}\right) & =5.4 \pm 0.2 \\
\dot{M} /\left(M_{\odot} / y r\right) & =(2.5 \pm 1.5) 10^{-5} \text { helium }+110^{-5} \text { hydrogen (estimated) } \\
V_{\text {rot }} \sin i /(\mathrm{km} / \mathrm{s}) & =450 \pm 100
\end{aligned}
$$

Special emphasis is put on the fit to the He I line at $4471 \AA$, which appears as an unshifted absorption profile of quasi "photospheric" origin. A striking result is the very high rotational velocity which is indicated by its profile (Fig. 3 ).

The position of this star in the HR-diagram is among the bulk of Tuminous association members, which are in the hydrogen burning phase. This result, together with the star's high rotational velocity, favours the scenario of rotationally induced mixing (Maeder, 1987) for the formation of this Wolf-Rayet star. 


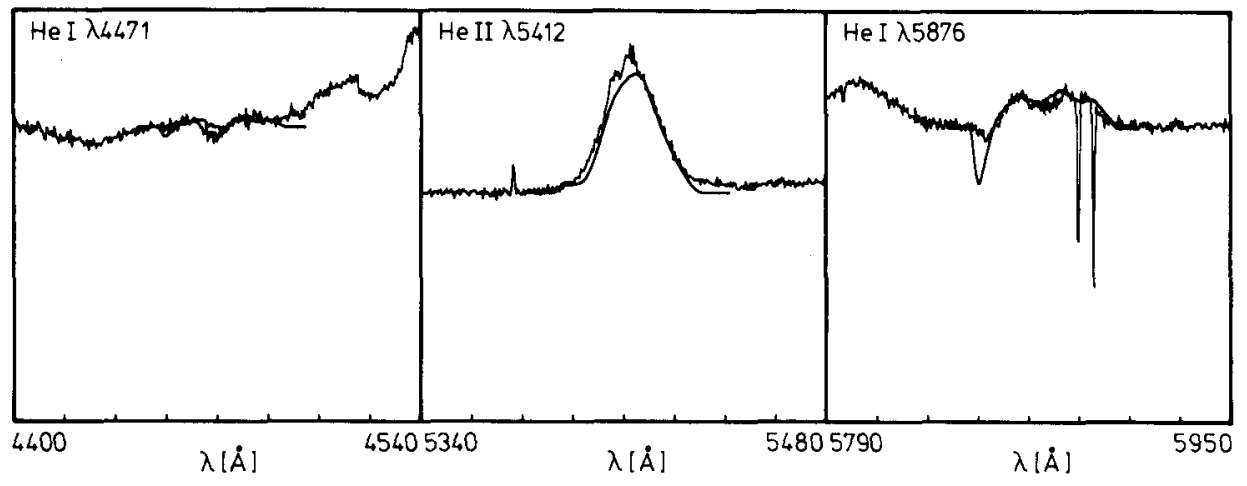

Figure 1. Theoretical line profiles of the final model with $\beta=0.5$ superimposed on the observations. The profiles are rotationally broadend by $450 \mathrm{~km} / \mathrm{s}$

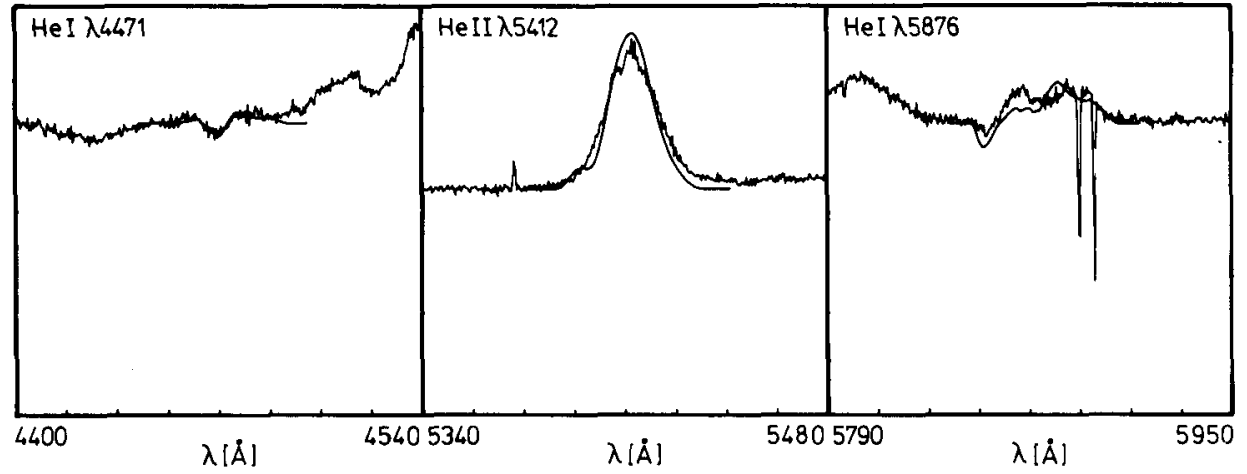

Figure 2. Same as Fig. 1, but for the final model obtained with $B=1$ and one additional free electron per helium atom

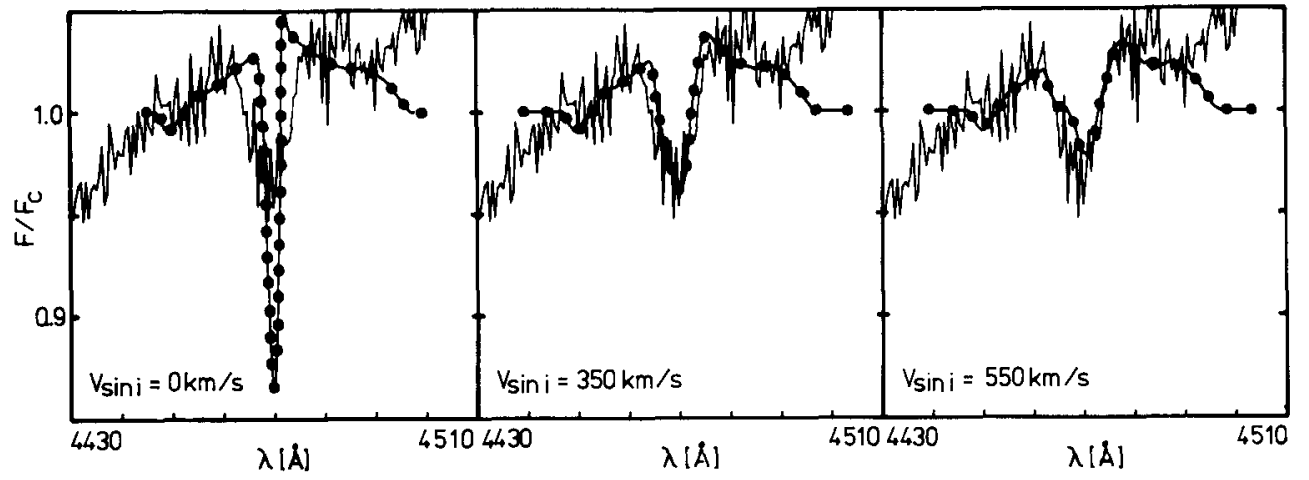

Figure 3. Rotational broadening of He I $\lambda 4471$, assuming angular momentum conservation. The best profile fit is obtained with $v_{\text {rot }} \sin i=450 \mathrm{~km} / \mathrm{s}$.

\section{REFERENCES}

Maeder,A.: 1987, Astron. Astrophys. 178, 159

Hamann,W.-R., Schmutz,W.: 1986, Astron. Astrophys. 174, 173

Wessolowski,U., Schmutz,W., Hamann,W.-R.: 1987, Astron. Astrophys. (in press) 